EPJ Web of Conferences 70, 00063 (2014)

DOI: $10.1051 /$ epjconf/ 20147000063

(C) Owned by the authors, published by EDP Sciences, 2014

\title{
CP violation in D-meson decays
}

\section{plenary talk at the 1st International Conference on New Frontiers in Physics}

\author{
M.I.Vysotsky ${ }^{1,2, a}$
}

${ }^{1}$ A.I. Alikhanov Institute of Theoretical and Experimental Physics, Moscow, 117259, Russia

${ }^{2}$ Novosibirsk State University, Novosibirsk, 630090, Russia

\begin{abstract}
Since most probably Standard Model cannot explain large value of CP asymmetries recently observed in $D$-meson decays the fourth quark-lepton generation explanation of it is proposed. As a byproduct weakly mixed leptons of the fourth generation enable to save the baryon number of the Universe from erasure by sphalerons.
\end{abstract}

\section{Standard Model}

The talk is based on the paper [1].

In $2011 \mathrm{LHCb}$ collaboration has measured the unexpectedly large $\mathrm{CP}$ violating asymmetries in $D \rightarrow \pi^{+} \pi^{-}$and $D \rightarrow K^{+} K^{-}$decays [2]:

$$
\left.\Delta A_{C P}^{L H C b} \equiv A_{C P}\left(K^{+} K^{-}\right)-A_{C P}\left(\pi^{+} \pi^{-}\right)=[-0.82 \pm 0.21 \text { (stat.) } \pm 0.11 \text { (syst. })\right] \% ，
$$

where

$$
A_{C P}\left(\pi^{+} \pi^{-}\right)=\frac{\Gamma\left(D^{0} \rightarrow \pi^{+} \pi^{-}\right)-\Gamma\left(\bar{D}^{0} \rightarrow \pi^{+} \pi^{-}\right)}{\Gamma\left(D^{0} \rightarrow \pi^{+} \pi^{-}\right)+\Gamma\left(\bar{D}^{0} \rightarrow \pi^{+} \pi^{-}\right)}
$$

and $A_{C P}\left(K^{+} K^{-}\right)$is defined analogously.

This result was later confirmed by CDF collaboration, which obtains [3]:

$$
\left.\Delta A_{C P}^{C D F}=[-0.62 \pm 0.21 \text { (stat.) } \pm 0.10 \text { (syst. })\right] \% \text {. }
$$

The following questions concerning experimental results (1) and (3) arises:

1. Whether in the Standard Model the CPV in these decays as large as $0.5 \%-1 \%$ can be obtained? Our answer is "most probably NO".

2. Is really $\left|\Delta A_{C P}\right|>0.5 \%$ ?

This should be clarified soon when all available data will be accounted for by LHCb collaboration.

3. Is there any New Physics that allows big CPV in $D$ decays?

Yes, and the 4'th quark-lepton generation is one of the examples of such New Physics.

In the Standard Model the CPV in $D(\bar{D}) \rightarrow \pi^{+} \pi^{-}$decays originates from the interference of the tree and penguin diagrams shown in Fig. 1. For $D(\bar{D}) \rightarrow K^{+} K^{-}$decays $d$-quarks in these diagrams should be substituted by $s$-quarks.

\footnotetext{
a e-mail: vysotsky@itep.ru
}

This is an Open Access article distributed under the terms of the Creative Commons Attribution License 2.0, which permits unrestricted use, distribution, and reproduction in any medium, provided the original work is properly cited. 


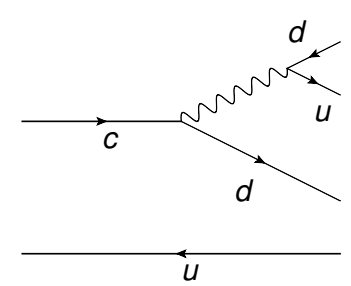

a)

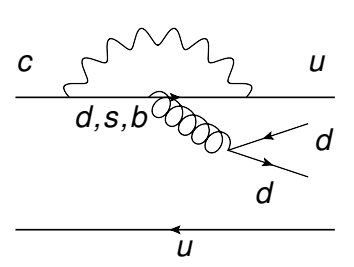

b)

Figure 1. Quark diagrams describing $D \longrightarrow \pi^{+} \pi^{-}$decay in the Standard Model. A wavy line denotes $W$-boson, a curly line - gluon.

It is convenient to present the penguin diagram contribution to $D \rightarrow \pi^{+} \pi^{-}$decay amplitude in the following form [4]:

$$
\begin{array}{r}
V_{c d} V_{u d}^{*} f\left(m_{d}\right)+V_{c s} V_{u s}^{*} f\left(m_{s}\right)+V_{c b} V_{u b}^{*} f\left(m_{b}\right)= \\
=V_{c d} V_{u d}^{*}\left[f\left(m_{d}\right)-f\left(m_{s}\right)\right]+V_{c b} V_{u b}^{*}\left[f\left(m_{b}\right)-f\left(m_{s}\right)\right],
\end{array}
$$

attributing the first term to the tree amplitude and considering the second term only as the penguin amplitude.

In the case of $D \rightarrow K^{+} K^{-}$decay the following presentation is useful [4]:

$$
\begin{array}{r}
V_{c d} V_{u d}^{*} f\left(m_{d}\right)+V_{c s} V_{u s}^{*} f\left(m_{s}\right)+V_{c b} V_{u b}^{*} f\left(m_{b}\right)= \\
=V_{c s} V_{u s}^{*}\left[f\left(m_{s}\right)-f\left(m_{d}\right)\right]+V_{c b} V_{u b}^{*}\left[f\left(m_{b}\right)-f\left(m_{d}\right)\right] ;
\end{array}
$$

the first term is attributed to the tree amplitude while the second one is the penguin amplitude.

Designating the absolute values of $D \rightarrow \pi^{+} \pi^{-}$decay amplitudes by $T$ and $P$ we get:

$$
\begin{aligned}
& A_{\pi^{+} \pi^{-}}=T\left[1+\frac{P}{T} e^{i(\delta-\gamma)}\right], \\
& \bar{A}_{\pi^{+} \pi^{-}}=T\left[1+\frac{P}{T} e^{i(\delta+\gamma)}\right],
\end{aligned}
$$

where $\delta$ stands for the difference of the strong interaction phases of tree and penguin amplitudes, while $\gamma \approx 70^{0}$ is the phase of $V_{u b}$ (the product $V_{c d} V_{u d}^{*}$ as well as $V_{c b}$ are practically real in the standard parametrization of CKM matrix).

From (6) for CPV asymmetry we obtain:

$$
A_{C P}\left(\pi^{+} \pi^{-}\right)=2 \frac{P}{T} \sin \delta \sin \gamma .
$$

Since $\sin \gamma$ is close to one we will substitute $\sin \gamma=1$ in the expression for asymmetry.

Let us present an argument demonstrating that $\delta$ also can be close to $90^{\circ}$. In $D \rightarrow \pi \pi$ decay rates tree diagram dominates, and corresponding to it 4-fermion Hamiltonian has parts with isospin $1 / 2$ and $3 / 2$. That is why the produced $\pi$-meson may have isospins zero or two. So three decay probabilities $\left(D^{+} \rightarrow \pi^{+} \pi^{0}, D^{0} \rightarrow \pi^{+} \pi^{-}\right.$and $D^{0} \rightarrow \pi^{0} \pi^{0}$ ) depend on decay amplitudes absolute values $A_{0}$ and $A_{2}$ and their strong phases difference $\delta_{0}-\delta_{2}$. From the experimentally measured branching ratios [5]:

$$
\operatorname{Br}\left(D^{+} \rightarrow \pi^{+} \pi^{0}\right)=[12.6 \pm 0.9] \cdot 10^{-4}, \operatorname{Br}\left(D^{0} \rightarrow \pi^{0} \pi^{0}\right)=[8.0 \pm 0.8] \cdot 10^{-4},
$$




$$
\operatorname{Br}\left(D^{0} \rightarrow \pi^{+} \pi^{-}\right)=[13.97 \pm 0.26] \cdot 10^{-4}
$$

for the difference of phases of amplitudes with $I=0$ and $I=2$ we get:

$$
\left|\delta_{0}-\delta_{2}\right|=86^{0} \pm 4^{0}
$$

In equation (7) $\delta$ stands for the difference of the strong phases of penguin amplitude which has $I=1 / 2$ and produces pions with $I=0$ and tree amplitude, which has parts with $I=1 / 2$ and $I=3 / 2$ and produces pions with $I=0$ and $I=2$, that is why $\delta \neq \delta_{0}-\delta_{2}$. Nevertheless (9) demonstrates that $\delta$ can be large, and in what follows we will substitute $\sin \delta=1$ in (7).

In the limit of $U$-spin ( $d \leftrightarrow s$ interchange) symmetry tree amplitude of $D(\bar{D}) \rightarrow K^{+} K^{-}$decay differs by a sign from that of $D(\bar{D}) \rightarrow \pi^{+} \pi^{-}$decay, while the penguin amplitudes of these decays are equal, that is why

$$
A_{C P}\left(K^{+} K^{-}\right)=-A_{C P}\left(\pi^{+} \pi^{-}\right) .
$$

Let us suppose that (10) is not badly violated, so finally we obtain:

$$
\Delta A_{C P}=4 \frac{P}{T}
$$

and let us try to understand if in the Standard Model we can obtain

$$
\frac{P}{T}=1.8 \cdot 10^{-3}
$$

which we need to reproduce an average value of the $\mathrm{LHCb}$ and $\mathrm{CDF}$ results.

The simple estimate gives:

$$
\frac{P}{T} \sim \frac{V_{c b} V_{u b}}{V_{c d}} \frac{\alpha_{s}\left(m_{c}\right)}{\pi} \approx 10^{-4},
$$

which is approximately 20 times smaller than the number which follows from experimental data.

Though the four-fermion quark hamiltonian responsible for these decays is known strong interactions prevent us from calculation of decay amplitudes. What can be done is an estimation of decay amplitudes with the help of factorization. Let us start from the tree diagram shown in Fig. 1a) which dominates in the decay amplitude:

$$
\begin{aligned}
T & =\frac{G_{F}}{\sqrt{2}} V_{c d}<\pi^{+} \pi^{-}\left|\bar{d} \gamma_{\alpha}\left(1+\gamma_{5}\right) c \bar{u} \gamma_{\alpha}\left(1+\gamma_{5}\right) d\right| D^{0}>\times \\
& \times\left\{\frac{2}{3}\left[\alpha_{s}\left(m_{c}\right) / \alpha_{s}\left(M_{W}\right)\right]^{-2 / b}+\frac{1}{3}\left[\alpha_{s}\left(m_{c}\right) / \alpha_{s}\left(M_{W}\right)\right]^{4 / b}\right\},
\end{aligned}
$$

where the last factor originates from the summation of the gluon exchanges in the leading logarithmic approximation. Substituting in it $b=11-2 / 3 N_{f}=23 / 3, \alpha_{s}\left(M_{W}\right)=0.12, \alpha_{s}\left(m_{c}\right)=0.3$ we find that it is close to one: \{\}$=1.1$. Factorizing decay amplitude we obtain:

$$
\begin{aligned}
T & =1.1 \frac{G_{F}}{\sqrt{2}} V_{c d}<\pi^{+}\left|\bar{u} \gamma_{\alpha}\left(1+\gamma_{5}\right) d\right| 0><\pi^{-}\left|\bar{d} \gamma_{\alpha}\left(1+\gamma_{5}\right) c\right| D^{0}>= \\
& =1.1 \frac{G_{F}}{\sqrt{2}} V_{c d} f_{\pi} k_{1 \alpha}\left[f_{+}^{\pi}(0)\left(p+k_{2}\right)_{\alpha}+f_{-}^{\pi}(0)\left(p-k_{2}\right)_{\alpha}\right]=1.1 \frac{G_{F}}{\sqrt{2}} V_{c d} f_{\pi} f_{+}^{\pi}(0) m_{D}^{2}
\end{aligned}
$$

where $k_{1}$ and $k_{2}$ are the momenta of the produced $\pi$-mesons, $p$ is $D$-meson momentum and we neglect $m_{\pi}^{2}$ in comparison with $m_{D}^{2}$. 
The value of $D^{0} \rightarrow \pi^{+} e^{+} v$ transition formfactor at $q^{2}=0$ can be found in [5]:

$$
f_{+}^{\pi}(0)\left|V_{c d}\right|=0.152 \pm 0.005, f_{+}^{\pi}(0)=0.66,
$$

and for the decay width we get:

$$
\Gamma_{D \rightarrow \pi^{+} \pi^{-}}^{\text {theor. }}=\frac{G_{F}^{2}}{2} \frac{\left(1.1 V_{c d} f_{+}^{\pi}(0) f_{\pi} m_{D}^{2}\right)^{2}}{16 \pi m_{D}}=6.2 \cdot 10^{9}\left(\mathrm{sec}^{-1}\right),
$$

where $f_{\pi}=130 \mathrm{MeV}$ was used.

From the branching ratio of $D^{0} \rightarrow \pi^{+} \pi^{-}$decay (8) and $D^{0}$-meson mean life $\tau_{D^{0}}=0.41 \cdot 10^{-12} \sec$ we get:

$$
\Gamma_{D \rightarrow \pi^{+} \pi^{-}}^{\exp }=3.4 \cdot 10^{9}\left(\mathrm{sec}^{-1}\right),
$$

So the naive factorization overestimates the decay amplitude by the factor $\sqrt{6.2 / 3.4} \approx 1.4$. We see that in the case of the tree diagram the accuracy of factorization approximation is very good.

The four-fermion QCD penguin amplitude which describes $D \rightarrow \pi^{+} \pi^{-}$decay looks like:

$$
\begin{aligned}
H(P) & =\frac{G_{F}}{\sqrt{2}} V_{c b} V_{u b}^{*} \frac{\alpha_{s}\left(m_{c}\right)}{12 \pi} \ln \left(\frac{m_{b}}{m_{c}}\right)^{2}\left(\bar{u} \gamma_{\alpha}\left(1+\gamma_{5}\right) \vec{\lambda} c\right)\left(\bar{d} \gamma_{\alpha} \vec{\lambda} d\right)= \\
& =\frac{G_{F}}{\sqrt{2}} V_{c b} V_{u b}^{*} \frac{\alpha_{s}\left(m_{c}\right)}{12 \pi} \ln \left(\frac{m_{b}}{m_{c}}\right)^{2}\left[\left(\bar{u} \gamma_{\alpha}\left(1+\gamma_{5}\right) d\right)\left(\bar{d} \gamma_{\alpha}\left(1+\gamma_{5}\right) c\right)-\right. \\
& \left.-2 \bar{u}\left(1-\gamma_{5}\right) d \bar{d}\left(1+\gamma_{5}\right) c\right] \frac{8}{9},
\end{aligned}
$$

where $\vec{\lambda}$ are the Gell-Mann SU(3) matrices and we use the Fierz identities $\vec{\lambda}_{a b} \vec{\lambda}_{c d}=-2 / 3 \delta_{a b} \delta_{c d}+$ $2 \delta_{a d} \delta_{b c}, \bar{\psi} \gamma_{\alpha}\left(1+\gamma_{5}\right) \varphi \bar{\chi} \gamma_{\alpha}\left(1+\gamma_{5}\right) \eta=\bar{\psi} \gamma_{\alpha}\left(1+\gamma_{5}\right) \eta \bar{\chi} \gamma_{\alpha}\left(1+\gamma_{5}\right) \varphi, \bar{\psi} \gamma_{\alpha}\left(1+\gamma_{5}\right) \varphi \bar{\chi} \gamma_{\alpha}\left(1-\gamma_{5}\right) \eta=-2 \bar{\psi}(1-$ $\left.\gamma_{5}\right) \eta \bar{\chi}\left(1+\gamma_{5}\right) \varphi$. Also the identity $<\pi^{+}\left|\bar{u}_{a} O d_{b}\right| 0>=1 / 3 \delta_{a b}<\pi^{+}|\bar{u} O d| 0>$, where $O \equiv \gamma_{\alpha} \gamma_{5}$ or $\gamma_{5}$ was used.

Calculating the matrix element in the factorization approximation with the help of the equations of motion for quark fields we get:

$$
P=\frac{G_{F}}{\sqrt{2}}\left|V_{c b} V_{u b}^{*}\right| \frac{\alpha_{s}\left(m_{c}\right)}{12 \pi} \ln \left(\frac{m_{b}}{m_{c}}\right)^{2} \frac{8}{9} f_{\pi} f_{+}^{\pi}(0) m_{D}^{2}\left[1+\frac{2 m_{\pi}^{2}}{m_{c}\left(m_{u}+m_{d}\right)}\right] .
$$

Dividing by the experimental value of the tree amplitude and using Eq.(15) we obtain:

$$
P / T=\frac{1.4}{1.1} \frac{8}{9} \frac{\left|V_{c b} V_{u b}^{*}\right|}{\left|V_{c d}\right|} \frac{\alpha_{s}\left(m_{c}\right)}{12 \pi} \ln \left(\frac{m_{b}}{m_{c}}\right)^{2}\left[1+\frac{2 m_{\pi}^{2}}{m_{c}\left(m_{u}+m_{d}\right)}\right] .
$$

Substituting $\left|V_{c d}\right|=0.23,\left|V_{u b}\right|=3.9 \cdot 10^{-3}, V_{c b}=41 \cdot 10^{-3}, \alpha_{s}\left(m_{c}\right)=0.3, m_{b}=4.5 \mathrm{GeV}, m_{c}=1.3$ $\mathrm{GeV}, m_{u}+m_{d}=6 \mathrm{MeV}$ we get:

$$
P / T \approx 9 \cdot 10^{-5} \text {. }
$$

Comparing with (12) we see that in order to fit the experimental result on $\Delta A_{C P}$ penguin amplitude should be enhanced by factor 20 in comparison with what factorization gives. Concerning tree amplitudes we found in this section that factorization result differs from the experimental value by factor 1.4 in the case of $D \rightarrow \pi^{+} \pi^{-}$decay.

Let us look now how accurate the factorization approximation to the penguin amplitudes in $B$ - and $K$-meson decays is. 


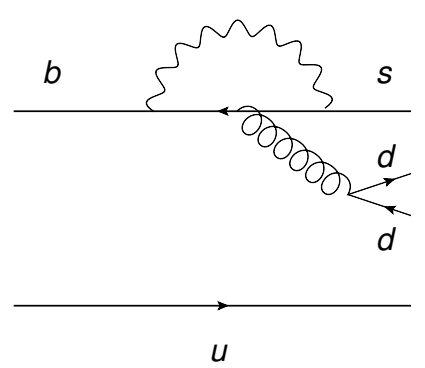

Figure 2. $B_{u} \rightarrow \pi^{+} K^{0}$ decay proceeds through the penguin amplitude only.

$B_{u} \rightarrow \pi^{+} K^{0}$ decay is described by the penguin amplitude shown in Fig. 2.

The Hamiltonian responsible for this decay looks like:

$$
\hat{H}=\frac{G_{F}}{\sqrt{2}} V_{t b} V_{t s}^{*}\left[c_{3} O_{3}+c_{4} O_{4}+c_{5} O_{5}+c_{6} O_{6}\right],
$$

$V_{t b} V_{t s}^{*}$ is substituted for $V_{c b} V_{c s^{*}}+V_{u b} V_{u s}^{*}$ and

$$
\begin{aligned}
O_{3} & =\bar{s} \gamma_{\alpha}\left(1+\gamma_{5}\right) b \bar{d} \gamma_{\alpha}\left(1+\gamma_{5}\right) d \\
O_{4} & =\bar{s}_{a} \gamma_{\alpha}\left(1+\gamma_{5}\right) b_{c} \bar{d}_{c} \gamma_{\alpha}\left(1+\gamma_{5}\right) d_{a} \\
O_{5} & =\bar{s} \gamma_{\alpha}\left(1+\gamma_{5}\right) b \bar{d} \gamma_{\alpha}\left(1-\gamma_{5}\right) d \\
O_{6} & =\bar{s}_{a} \gamma_{\alpha}\left(1+\gamma_{5}\right) b_{c} \bar{d}_{c} \gamma_{\alpha}\left(1-\gamma_{5}\right) d_{a},
\end{aligned}
$$

where $a, c=1,2,3$ are the color indexes.

Using the Fierz identities as well as $<K^{0}\left|\bar{s}_{a} O d_{b}\right| 0>=\frac{1}{3} \delta_{a b}<K^{0}|\bar{s} O d| 0>$ identity we get:

$$
\hat{H}=\frac{G_{F}}{\sqrt{2}} V_{t b} V_{t s}^{*}\left[a_{4} \bar{s} \gamma_{\alpha}\left(1+\gamma_{5}\right) d \bar{d} \gamma_{\alpha}\left(1+\gamma_{5}\right) b-2 a_{6} \bar{s}\left(1-\gamma_{5}\right) d \bar{d}\left(1+\gamma_{5}\right) b\right]
$$

where $a_{4}=\frac{1}{3} c_{3}+c_{4}, a_{6}=\frac{1}{3} c_{5}+c_{6}$. Calculating the matrix element in the factorization approximation we obtain:

$$
M=\frac{G_{F}}{\sqrt{2}} V_{t b} V_{t s}^{*} f_{K} f_{+}(0) m_{B}^{2}\left[a_{4}+a_{6} \frac{2 m_{K}^{2}}{m_{b} m_{s}}\right],
$$

where in the leading logarithmic approximation the following approximate equation is valid:

$$
a_{4}=a_{6}=-\frac{\alpha_{s}\left(m_{b}\right)}{12 \pi} \ln \left(\frac{M_{W}}{m_{b}}\right)^{2} \approx-0.03,
$$

while at NLO approximation from Table 1 of [6] we obtain: $a_{4}=-0.031, a_{6}=-0.042$. Substituting $m_{s}=100 \mathrm{MeV}, m_{b}=4.5 \mathrm{GeV}$ we get:

$$
\Gamma_{\left(B_{u} \rightarrow \pi^{+} K^{0}\right)}^{\mathrm{fact}}=\frac{G_{F}^{2}\left|V_{t s}\right|^{2}}{32 \pi} f_{K}^{2} f_{+}^{2}(0) m_{B}^{3}(0.076)^{2}=4.1 \cdot 10^{6}\left(\mathrm{sec}^{-1}\right),
$$

where $V_{t s}=39 \cdot 10^{-3}$ and $f_{+}(0)=0.25$ from [5] was used. The experimental result is:

$$
\Gamma_{\left(B_{u} \rightarrow \pi^{+} K^{0}\right)}^{\exp }=14 \cdot 10^{6}\left(\sec ^{-1}\right),
$$


So, the factorization result is enhanced by the factor

$$
P / P_{\text {fact }}=\sqrt{14 / 4.1}=1.8
$$

The numerical value of the penguin amplitude is important in the calculation of $\mathrm{CP}$ asymmetries in $B \rightarrow \pi K$ and $B \rightarrow \pi \pi$ decays [7].

$s \rightarrow d$ penguin transition changes the isospin by $1 / 2$ in this way explaining a famous $\Delta I=1 / 2$ rule in $K \rightarrow \pi \pi$ decays. The calculation of $K_{S} \rightarrow \pi^{+} \pi^{-}$decay amplitude generated by a penguin transition using the factorization underestimates the amplitude by the factor 2-3 according to [6,8].

In view of the results for $B$ and $K$ decays we can cautiously suppose that for $D \rightarrow \pi^{+} \pi^{-}$decay factorization calculation underestimate the penguin amplitude by factor 5 at most leading to:

$$
\triangle A_{C P}^{\text {theor }} \lesssim 0.2 \%
$$

The following alternative emerges: the experimental results are wrong or New Physics is found. (To be objective let us cite a paper [9] which state that in framework of Standard Model large CPV in $D$ decays can be explained.) Of course we cannot determine what kind of new particles and interactions are responsible for large CPV asymmetry in $D \rightarrow \pi^{+} \pi^{-}\left(K^{+} K^{-}\right)$decays. However, in the next section we will propose the straightforward generalization of the Standard Model in which large CPV in $D$ decays can be explained.

\section{The fourth generation: enhancement of CPV in $D$ decays}

As it was stated in paper [10] the introduction of the fourth quark-lepton generation may easily remove Standard Model upper bound (31) matching the experimental results [2, 3]. In the case of the fourth generation the additional term with the intermediate $b^{\prime}$ quark should be added to the expression for the penguin amplitude. In this way expression (4) is substituted by:

$$
\begin{array}{r}
V_{c d} V_{u d}^{*} f\left(m_{d}\right)+V_{c s} V_{u s}^{*} f\left(m_{s}\right)+V_{c b} V_{u b}^{*} f\left(m_{b}\right)+V_{c b^{\prime}} V_{u b^{\prime}} f\left(m_{b^{\prime}}\right)= \\
=V_{c d} V_{u d}^{*}\left[f\left(m_{d}\right)-f\left(m_{s}\right)\right]+V_{c b} V_{u b}^{*}\left[f\left(m_{b}\right)-f\left(m_{s}\right)\right]+V_{c b^{\prime}} V_{u b^{\prime}}\left[f\left(m_{b^{\prime}}\right)-f\left(m_{s}\right)\right],
\end{array}
$$

where the unitarity of $4 \times 4$ quark mixing matrix is used. According to the experimental constraints from the direct searches of the fourth generation quarks $b^{\prime}$ should weigh several hundreds $\mathrm{GeV}$, that is why $f\left(m_{b^{\prime}}\right)$ is small and can be neglected just as it is done with $t$-quark contribution to $b \rightarrow s$ penguin, see the remark after Eq. (23). In order to enhance SM contribution to the penguin amplitude we should suppose that the term $V_{c b^{\prime}} V_{u b^{\prime}} f\left(m_{s}\right)$ dominates in it.

Then the enhancement of $A_{C P}$ in the case of the fourth generation is equal to:

$$
\begin{aligned}
\frac{P_{4}}{P_{S M}} & =\frac{\ln \left(m_{W} / m_{c}\right)}{\ln \left(m_{b} / m_{c}\right)} \frac{\mid V_{c b^{\prime}} V_{u b^{\prime}}^{*}}{\left|V_{c b} V_{u b}\right|} \frac{\sin \left(\arg V_{c b^{\prime}} V_{u b^{\prime}}^{*}\right)}{\sin \gamma} \approx \\
& \approx 3.3 \frac{3 \cdot 10^{-4}}{1.5 \cdot 10^{-4}} \approx 6,
\end{aligned}
$$

where in the last equality we use the results on the allowed values of the product $\left|V_{c b^{\prime}} V_{u b^{\prime}}^{*}\right| \sin \left(\arg V_{c b^{\prime}} V_{u b^{\prime}}\right)$ from Fig. 1 of paper [11]. So we see that the enhancement necessary to describe experimental data on $\Delta A_{C P}$ can be achieved in the case of the fourth generation. 


\section{Saving baryon number by a long-lived fourth generation neutrino}

If weakly mixed particles exist, then the sphaleron processes can create the baryon asymmetry of the Universe [12]. As it was noted in [13] the long-lived fourth generation particles save baryon asymmetry generated at the Early Universe from erasure by the sphaleron transitions. The sphaleron transitions conserve $B-L$, that is why if at early universe $B_{0}=L_{0} \neq 0$ is generated, then the final baryon and lepton asymmetries being proportional to $B-L$ are completely erased. If the fourth generation particles weakly mix with three quark-lepton generations of Standard Model, then two additional quantities are conserved: $B_{4}-L_{4}$ and $L-3 L_{4}$, where $B_{4}$ and $L_{4}$ are the densities of baryons and leptons of the fourth generation, while $B$ and $L$ are the densities of baryons and leptons of three light generations. In [13] initial asymmetries $B_{0}=L_{0}=3 \Delta, B_{4}^{0}=L_{4}^{0}=0$ were chosen and since $L-3 L_{4}=3 \Delta \neq 0$ the $B+B_{4}$ the number density at the sphaleron freeze-out temperature being proportional to the linear superposition of conserved quantities is nonzero. After sphaleron freeze-out $B+B_{4}$ is conserved and equals to the modern baryon density of the Universe up to the factor which takes into account the general expansion ${ }^{1}$.

For such a scenario to occur the lifetimes of the fourth generation quarks and leptons should be larger than the lifetime of the Universe at the sphaleron freeze-out: $\tau_{4}>M_{\mathrm{Pl}} / T_{\mathrm{sph}}^{2} \sim 10^{-10} \mathrm{sec}$. For mixing angles in case of $b^{\prime} \rightarrow(c, u) W$ decay it gives $\theta<10^{-8}$ [13], much smaller than what we need to explain large CPV in $D$-decays, see Eq. (33).

So in our case the quarks of the fourth generation are too strongly mixed with the quarks of three light generations. However, let us suppose that the leptons of the fourth generation are weakly mixed with the leptons of three light generations. Let us introduce the baryon density $B^{\prime} \equiv B+B_{4}$ and take the initial conditions analogous to those in [13]: $B_{0}^{\prime}=L_{0}=3 \Delta, L_{4}^{0}=0$. As four independent chemical potentials we can choose: $\mu_{u_{L}}, \mu_{W}, \mu_{N_{L}}$ and $\mu \equiv \mu_{v_{e}}+\mu_{v_{\mu}}+\mu_{v_{\tau}}$, which are the chemical potentials for the upper type quarks, $W$-bosons, $4 G$ neutrino and sum over all SM neutrino chemical potentials. In the limit $\mu_{i} / T \ll 1$ the baryon and lepton densities are linear combinations of these chemical potentials with the coefficients which depend on the ratio of masses of the corresponding particles to the temperature. We will take into account the masses of $W$-boson, $t$-quark, $t^{\prime}$ - and $b^{\prime}$-quarks of the fourth generation and the fourth generation leptons $N$ and $E$, the masses of all the other components of primordial plasma can be neglected in comparison with $T_{\mathrm{sph}}$.

Finally we have four equations for four unknown chemical potentials: two quantities are conserved under the sphaleron transitions; we can choose them as

$$
\begin{aligned}
B^{\prime}-L-L_{4} & =0, \\
L-3 L_{4} & =3 \Delta .
\end{aligned}
$$

The third equation is that of neutrality of primordial plasma $Q=0$ and, finally, the sum of the chemical potentials of all the particles which are converted into nothing by sphaleron (qqql of each generation) equals zero. The value of masses of 4 generation particles we take from paper [14] in which the fit to the electroweak observables for higgs mass $m_{H}=125 \mathrm{GeV}$ was performed and recent LHC bounds on the masses of $t^{\prime}$ - and $b^{\prime}$-quarks were taken into account:

$$
\begin{aligned}
& m_{t^{\prime}}=634 \mathrm{GeV} \quad, \quad m_{b^{\prime}}=600 \mathrm{GeV}, \\
& m_{E}=107.6 \mathrm{GeV} \quad, \quad m_{N}=57.8 \mathrm{GeV} .
\end{aligned}
$$

A dashed blue line in Fig. 3 corresponds to the case of the unmixed fourth generation particles considered in [13]. The results for the case of the strongly mixed fourth generation quarks and the

\footnotetext{
${ }^{1}$ Let us note that if the fourth generation quarks decay after BBN the resulting abundances of light nucleus would differ from those in three generations case.
} 


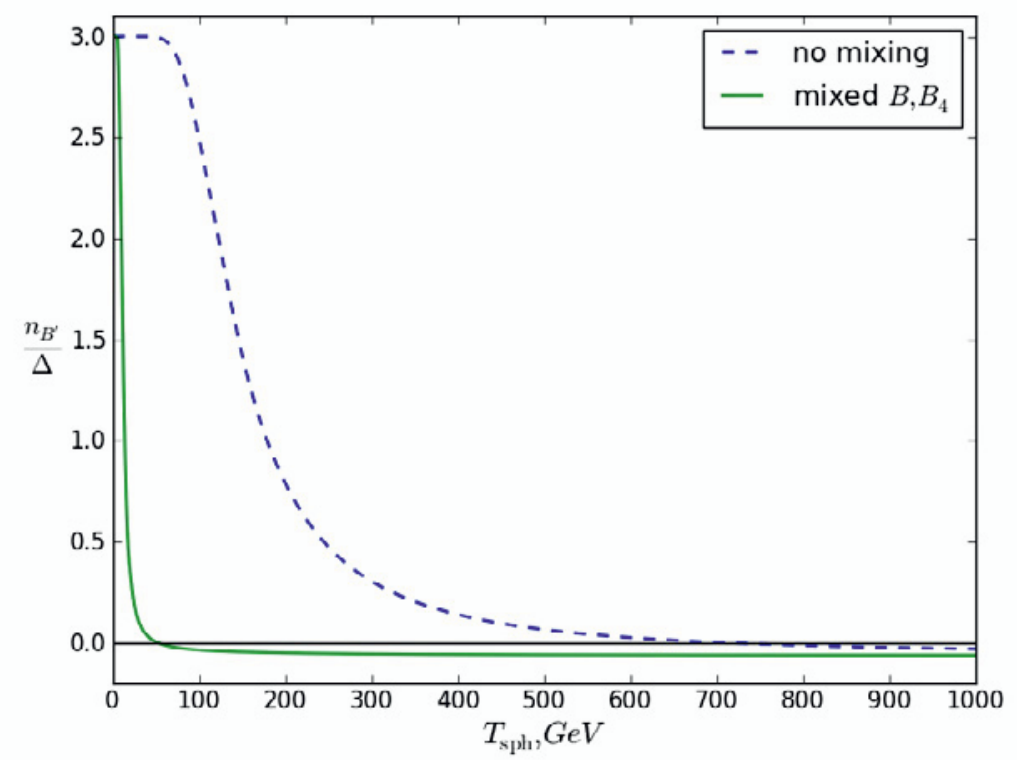

Figure 3. The final baryon asymmetry versus the initial asymmetry $n_{B^{\prime}} / \Delta$ as a function of sphaleron freeze-out temperature $T_{\text {sph }}(\mathrm{GeV})$ for the unmixed fourth generation is shown by a dashed (blue) line. It is analogous to Fig. 2 from [13] but for $m_{N}=57.8 \mathrm{GeV}, m_{E}=107.6 \mathrm{GeV}, m_{t^{\prime}}=634 \mathrm{GeV}, m_{b^{\prime}}=600 \mathrm{GeV}$. The final baryon asymmetry for the case of the mixed fourth generation quarks and the unmixed fourth generation leptons is shown by a solid (green) line.

unmixed fourth generation leptons are shown by a solid green line. In order for $N$ not to decay before sphaleron freeze-out which happens at $t_{U} \sim 10^{-10} \mathrm{sec}$ the mixing angles of $N$ with three light neutrinos should be small: $\theta_{i}<10^{-5}$ ( $N$ decays through four fermion interaction). A similar bound $\theta<10^{-6}$ comes from the search of $N$ at LEP II [15]. From Fig. 3 and Eq. (34) it follows that at sphaleron freeze-out $L_{4}$ is much larger than $B^{\prime}$, so $N$ should decay well before the nonrelativistic dark matter particles start to dominate in the energy density of the Universe, $\tau \sim 10^{13} \mathrm{sec}$, from which we obtain the lower bound $\theta>10^{-16}$. Let us note that direct searches exclude $N$ as a dark matter candidate [16].

In the limit $T_{\mathrm{sph}} \rightarrow 0$ heavy particles of the fourth generation are not produced: $B_{4}=L_{4}=0$, $B^{\prime}=L=3 \Delta$. In the physically interesting opposite limit $T_{\text {sph }} \gg m_{N}$ the value of baryon asymmetry is nonzero since the right-handed neutrinos of three light generations are not produced in the primordial plasma violating symmetry between the leptons of four generations which would occur at $T \gg m_{N}$. The characteristic time of the right-handed neutrino to thermalize is $T / m_{v}^{2}$ and for $m_{v} \lesssim 1 \mathrm{keV}$ (which is valid for three light neutrinos) this time is bigger than the lifetime of the Universe $t_{u}=M_{\mathrm{Pl}} / T^{2}$ for $T=T_{\text {sph }} \approx 200 \mathrm{GeV}[12]$.

\section{Conclusions}

We determine what value of the ratio of penguin to tree amplitudes of $D \rightarrow \pi^{+} \pi^{-}$decay is needed to get the observed CP asymmetry. In Section 1 we found that the factorization describes the tree amplitude 
with good accuracy; concerning the penguin amplitude it appears to be twenty times smaller than one needs to describe the experimental data on $A_{C P}$. We demonstrate that in the case of $B \rightarrow \pi^{+} K^{0}$ decay the factorization calculation underestimates the penguin amplitude by factor 2 . In the case of $K_{S} \rightarrow \pi^{+} \pi^{-}$decay penguin amplitude is enhanced by factor 2-3 in comparison with the factorization result.

Thus if confirmed on larger statistics and future systematics the result (1) demands New Physics.

In Section 2 we demonstrate that the fourth quark-lepton generation may enhance the penguin amplitude describing the experimental data. If the leptons of the fourth generation weakly mix with three light generation leptons, then the baryonic charge generated at high scale escapes the erasure by sphalerons and survive till now according to the results presented in Section 3.

I am grateful to my coauthors Alexander Dolgov, Sergey Godunov and Alexander Rozanov for fruitful collaboration.

I am grateful to the organizers of a stimulating conference in a great place.

I acknowledge the support of the grants RFBR 11-02-00441, 12-02-00193, the grant of the Russian Federation government 11.G34.31.0047, and the grant NSh-3172.2012.2.

\section{References}

[1] A.D.Dolgov, S.I.Godunov, A.N.Rozanov, M.I.Vysotsky, Pis'ma v ZhETF 96320 (2012);

[2] R. Aaij et al., Phys. Rev. Lett. 108111602 (2012).

[3] G. Punzi et al., Note 10784 (2012).

[4] G. Isisdori, J. Kamenik, Z. Ligeti, G. Perez, Phys. Lett. B 71146 (2012).

[5] K. Nakamura et al. (Particle Data Group), J. Phys. G 37075021 (2010).

[6] A.J. Buras et al., Nucl. Phys. B 37069 (1992).

[7] A.B. Kaidalov, M.I. Vysotsky, Phys. Atom. Nucl. 722126 (2009).

[8] A. Vainshtein, hep-ph/9906263 (1999).

[9] J. Brod, A.L. Kogan, J. Zupan, Phys.Rev. D 86014023 (2012).

[10] A.N. Rozanov, M.I. Vysotsky, Pis'ma v ZhETF 95443 (2012).

[11] S. Nandi, A. Soni, Phys. Rev. D 83114510 (2011).

[12] K. Dick, M. Lindner, M. Ratz, D. Wright, Phys. Rev. Lett. 844039 (2000).

[13] H. Murayama, V. Rentala, J. Shu, T. Yanagida, Phys. Lett. B 705208 (2011).

[14] O. Eberhardt et al., Phys.Rev. D 86013011 (2012).

[15] K. Ackerstaff et al., Eur. Phys. J. C1 45 (1998).

[16] H.-S. Lee, Z. Liu, A. Soni, Phys.Lett. B 70430 (2011).

[17] D.S. Gorbunov, V.A. Rubakov, Vvedenie v theoriyu rannei Vselennoi, URSS, Moskva, 2008. 